\title{
On Informative Objects
}

\author{
Niel Bezrookove
}

February 14, 2021

The following is a series of notes elaborating on some of the concepts of "Seepage in Objects: A Primer," a previous paper.

In "Seepage in Objects: A Primer (Bezrookove, 2020)," I defined objects as consisting not of matter or energy, but rather information arranged in a particular organization. The material of an object need not change in order for it to be informative, nor reveal new properties when organized differently. My first example was water, and I argued that we should not define water as merely a bundle of $\mathrm{H} 20$ objects with various properties but rather as strictly an organization of H20 objects that informs us of its properties when we encounter it. A change in its organization is informative to us, we're less uncertain of what we're dealing with and gain insight into the organization of what we denote as water.

My second example was a mechanical computer made of dominoes, which purely through changes in the organization of physical dominoes could produce informative state changes: computation. The dominoes themselves do not experience a material change when the sate of the computer changes - the entire substance of the domino computer, that which separates it from merely a bundle of dominoes, is not a property of the dominoes. Rather it is the organization of those dominoes that makes computation possible, and what the object of the 
domino computer consists of is purely that organization. An object therefore is entirely an emergent property, and to speak of any object as anything besides emergent is not only inconsistent with physical fact but also with how we interact with an object - ever-evolving our impressions, discovering new properties, learning more about what it is and could be.

This is not a completely novel ontology of objects. Rather, it is a restatement which reconsiders what I call "naïve ontology" and questions the traditional cleaving of objects between their matter and their form, and how they appear and what they substantively are. Of importance is the following from Aristotle's Metaphysics, in which the bifurcation of matter and form are presented as a viable solution to a difficult problem:

And in general "man" will be not one, but two things - "animal" and "two-footed." Evidently if we proceed in this way, as it is usual to define and explain, it will be impossible to answer and solve the difficulty. But if, as we maintain, man is part matter and part form - the matter being potentially, and the form actually man-, the point which we are investigating will no longer seem to be a difficulty. (1045 $\alpha$ ) (Aristotle, 350BCE)

If we define the matter itself as informative, we may believe that matter always presents itself to us in some kind of organization. We understand this organization within a space, perhaps all space, within a physical time, and it is this organization in space of objects that provides it with a sense of actuality that separates it from merely 'loose' matter like air or emptiness. We engage with this matter when we encounter it, and in this encounter we learn of the properties it possesses by virtue of the organization of its constituent matter. It is the information we gather from that which lends this matter its form, and every encounter gives us an understanding of its potentialities. Form here is what is informative about the organization of matter, and subsequently becomes what is meaningful about the object in question. Thusly it is its organization that gives the matter what Aristotle referred to as its form: 
Further, matter exists potentially, because it may attain to the form; but when it exists actually, it is then in the form. The same applies in all other cases, including those where the end is motion. (1050 $\alpha$ ) (Aristotle, 350BCE)

You can break a man into his constituent parts down to singular atoms, but it is all of these atoms and only these atoms, in only one specific organization and configuration in space, that strikes us as informative and different. And it is this information differentiating it from everything else in space that allows us to say we know of a man. Aristotle hints at this with the distinction between "potentiality and actuality," where 'motion' in Ancient Greek philosophy is synonymous with change as influenced by Heraclitus ${ }^{1}$ :

The term "actuality," with its implication of "complete reality," has been extended from motions, to which it properly belongs, to other things; for it is agreed that actuality is properly motion. (1047 $\alpha$ ) (Aristotle, 350BCE)

In this definition of objects, physical presence is justly not elevated to the primary thing of significance in defining object ontology. The question of what separates a 'real' thing from a 'fake' (imaginary) thing need not concern us; what separates them is the organization of the physical substances in question, and what they inform us of. Speaking of Russell's 'round square' (Russell, 1905), we may commit to the proposition that this object does indeed exist. However, there is no organization of physical substance possible that will inform us of the existence of a 'round square.' We can never speak of encountering it, it is completely non-informative as an object even though it is for the purpose of illustration in a philosophical argument genuinely meaningful. Russell insists that a 'round square' denotes nothing, but denoting nothing doesn't quite capture why the 'round square' as an object is still meaningful enough to be of value in a philosophical paper. To speak of a 'round square' is not making a mistaken judgement about the 'real' and 'unreal' (quoting MacColl through Russell), but

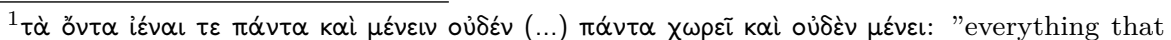

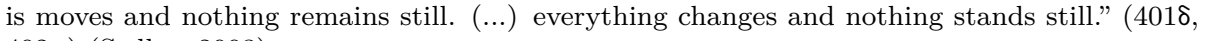
$402 \alpha$ ) (Sedley, 2003)
} 
an object which has no properties because nothing informative is possible with respect to it.

An objection might follow. I stated that the 'round square' is non-informative, yet nevertheless meaningful. However, I took this distinction for granted. How can we assume that if objects can be meaningful, they can also be non-informative? Suppose we have two computer terminals communicating with each other using an object such as a signal:

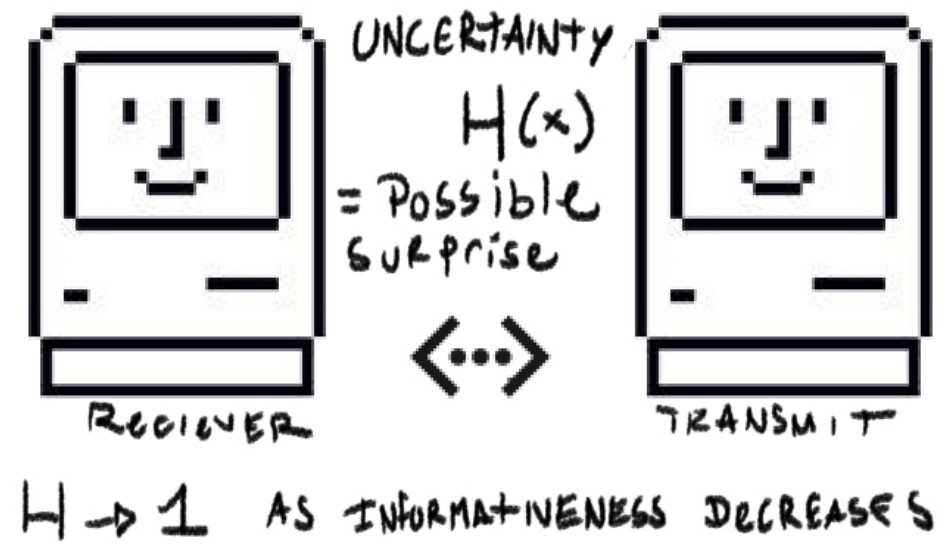

Extrapolating from Shannon's paper on communication (Shannon, 1948), we may define information as that which allows us to pick one message out of the range of possible messages from a channel of communication. Information reduces our uncertainty about the signal we're receiving. As we engage with an object, we become less uncertain about its properties - this is a meaningful interaction.

Shannon does address meaning: messages of meaning "refer to or are correlated according to some system with certain physical or conceptual entities." However the bulk of Shannon's paper deals strictly with what it means for something to be informative, and this need not be meaningful necessarily. However, our interest here is in how the informative potential of objects relates to a meaningful understanding of their properties. 
Practically, we cannot speak of an event between two computer terminals which can be said to be strictly both non-informative and meaningless ${ }^{2}$. Two possible edge case come to mind: a purely non-informative message, and a message yet to arrive.

Firstly, suppose the transmitting terminal is sending non-informative data ${ }^{3}$ to the receiving terminal. Uncertainty about the message doesn't change, but it does signal something meaningful to the receiving terminal which might solicit a request for a repetition of the message. In this particular sense, the communication is not informative (not reducing uncertainty about the message) but is nevertheless meaningful as it tells us something about the signal we're receiving. One can apply this to a conversation as well: even a purely incoherent statement can give us a meaningful idea about what we're hearing. Maybe we're hearing an unfamiliar language, or there's a problem somewhere in the communication channel. A meaningless message is in this strict sense impossible, even if it is non-informative. Along the same lines, interacting with an object is always meaningful even in exceptionally rare cases where our interaction results in absolutely nothing informative.

Secondly, suppose we await a transmission by a transmitter which we've yet to receive. Is the message informative? We're unable to answer that question. Yet this pause is meaningful, as it perhaps tells us something about the latency of the signal. Even though we've yet to learn something about the message, something about its properties appears to be revealed.

Therefore, it is impossible for an object to be meaningless, to reveal nothing even though our engagement with it might very well be non-informative. Every encounter with an object, even non-existent objects and non-informative objects

\footnotetext{
${ }^{2}$ An argument that 'meaninglessness' is itself an incoherent concept can certainly be made, but now's not the time

${ }^{3} \mathrm{~A}$ message entirely random so that no message is more probable than another and we learn nothing
} 
like the 'round square' leaves us with an impression that makes that object meaningful in an otherwise meaningless mist of floating matter and thought.

\section{References}

Russell, B. (1905). On denoting. Mind, 56(14), 479-493.

Shannon, C. E. (1948). A mathematical theory of communication. Bell Syst. Tech. J., 27(3), 379-423. http://dblp.uni-trier.de/db/journals/bstj/ bstj27.html\#Shannon48

Sedley, D. (2003). Plato's cratylus. Cambridge University Press. https://doi. org/10.1017/CBO9780511482649

Bezrookove, N. (2020). Seepage in objects: A primer. osf.io/preprints/socarxiv/ hga6y

Aristotle. (350BCE). Metaphysics (T. by W. D. Ross, Ed.). The Internet Classics Archive. http://classics.mit.edu/Aristotle/metaphysics.html 